\title{
Second-order wave diffraction by a circular cylinder using scaled boundary finite element method
}

\author{
H Song ${ }^{1}$ and L Tao ${ }^{2}$ \\ ${ }^{1}$ Griffith School of Engineering, Gold Coast campus, Griffith University, QLD4222, Australia \\ E-mail: h.song@griffith.edu.au \\ 2 School of Marine Science and Technology, Newcastle University, NE1 7RU, England, UK \\ E-mail: 1.tao@newcastle.ac.uk
}

\begin{abstract}
The scaled boundary finite element method (SBFEM) has achieved remarkable success in structural mechanics and fluid mechanics, combing the advantage of both FEM and BEM. Most of the previous works focus on linear problems, in which superposition principle is applicable. However, many physical problems in the real world are nonlinear and are described by nonlinear equations, challenging the application of the existing SBFEM model. A popular idea to solve a nonlinear problem is decomposing the nonlinear equation to a number of linear equations, and then solves them individually. In this paper, second-order wave diffraction by a circular cylinder is solved by SBFEM. By splitting the forcing term into two parts, the physical problem is described as two second-order boundary-value problems with different asymptotic behaviour at infinity. Expressing the velocity potentials as a series of depth-eigenfunctions, both of the 3D boundary-value problems are decomposed to a number of $2 \mathrm{D}$ boundary-value sub-problems, which are solved semi-analytically by SBFEM. Only the cylinder boundary is discretised with 1D curved finite-elements on the circumference of the cylinder, while the radial differential equation is solved completely analytically. The method can be extended to solve more complex wave-structure interaction problems resulting in direct engineering applications.
\end{abstract}

\section{Introduction}

Recently, a new semi-analytical method, namely scaled boundary finite element method has been successfully applied to many engineering problems, combining the advantages of both finite element method (FEM) and boundary element method (BEM) [10]. The method only discretises the body boundary surface with finite elements, then transforms the governing partial differential equations to ordinary matrix differential equations in radial direction which are solved analytically. The method solves the problems of singularities more accurately and the problems of unbounded domains more efficiently compared to FEM. Unlike BEM, it does not require a fundamental solution and is free from the difficulties caused by irregular frequencies and sharp corners. Fewer elements are needed to obtain very accurate solution. SBFEM was first proposed to solve the problems in soil-structure interaction by Song and Wolf [6], and later was applied to a variety of engineering fields. For example, Ekevid and Wiberg applied SBFEM and FEM to analyse wave propagation related to moving loads in railway engineering, demonstrating the performance of the method by presenting the dynamical response of a railroad section [2]. Teng et al. used SBFEM to simulate the water sloshing in a rectangular water container, finding that the SBFEM method gives much better results than the FEM method in the case of same 
mesh size [9]. By coupling SBFEM and FEM, Li et al. obtained semi-analytical solution for the characteristics of a two-dimensional dam-reservoir system with absorptive reservoir bottom in the frequency domain [3]. For short-crested waves diffracted by a vertical circular cylinder, Tao et al. obtained a high accurate and efficient result in unbounded domain by SBFEM with a small number of surface finite elements [8]. Song et al. extended their solution to water wave interaction with multiple cylinders of arbitrary shape recently [7].

However, most of the previous works focus on linear problems, in which superposition principle is applicable. In fact, many physical problems in the real world are nonlinear and are described by nonlinear equations, challenging the application of the existing SBFEM model. In this paper, second-order wave diffraction by a circular cylinder is solved by SBFEM. As an important attempt of the application of SBFEM in nonlinear dynamics, the method in this paper has a promising extension to solve more complex wave-structure interaction problems resulting in direct engineering applications.

\section{Theoretical consideration}

\subsection{General governing equations}

The governing equation of the wave diffracted by a circular cylinder is Laplace equation. By separating the variables in direction of the water depth, the governing equations to be solved are homogeneous Helmholtz equation and non-homogeneous Helmholtz equation. The equation can be expressed in a general form

$$
\nabla^{2} \phi(x, y)+k^{2} \phi(x, y)+f=0, \quad \text { in } \Omega,
$$

where $\nabla$ is the horizontal Laplacian, $\phi(x, y)$ is the velocity potential to be solved, $k$ is the wave number, $f$ is a known item, and $\Omega$ is the solution domain. Defining the Dirichlet boundary and Neumann boundary as $\Gamma_{\phi}$ and $\Gamma_{v}$ respectively, we have

$$
\begin{array}{rrr}
\phi=\bar{\phi}, & \text { on } & \Gamma_{\phi}, \\
\phi_{, n}=\bar{\phi}_{n}, & \text { on } & \Gamma_{v},
\end{array}
$$

where $\bar{\phi}_{n}$ is the prescribed normal derivative of the velocity potential, the overbar denotes a prescribed value, and comma in the subscript designates the partial derivative with respect to the variable following the comma.

\subsection{Scaled boundary finite-element transformation}

The finite-element method requires the weighted residuals of the governing equation to be zero. Hence Eqs. (1), (2) and (3) are multiplied by a weighting function $w$ and integrated over the solution domain and the boundary. Performing integration by parts, the resulting equation becomes

$$
\int_{\Omega} \nabla^{T} w \nabla \phi d \Omega-\int_{\Omega} w f d \Omega-\oint_{\Gamma_{v}} w \bar{\phi}_{n} d \Gamma=0 .
$$

SBFEM defines the solution domain $\Omega$ by scaling a single piecewise-smooth curve $S$ relative to a scaling centre $\left(x_{0}, y_{0}\right)$ (see Fig. 1). The circumferential coordinate $s$ is anticlockwise along the curve $S$ and the normalised radial coordinate $\xi$ is a scaling factor, defined as 1 at curve $S$ and 0 at the scaling centre. The whole solution domain $\Omega$ is in the range of $\xi_{0} \leq \xi \leq \xi_{1}$ and $s_{0} \leq s \leq s_{1}$. The two straight sections $s=s_{0}$ and $s=s_{1}$ are called side-faces. They coincide, if the curve $S$ is closed. For bounded domain, $\xi_{0}=0$ and $\xi_{1}=1$; whereas, for unbounded domain, $\xi_{0}=1$ and $\xi_{1}=\infty$. Therefore the Cartesian coordinates are transformed to the scaled boundary coordinates $\xi$ and $s$ with the scaling equations

$$
x=x_{0}+\xi x_{s}(s), \quad y=y_{0}+\xi y_{s}(s) .
$$




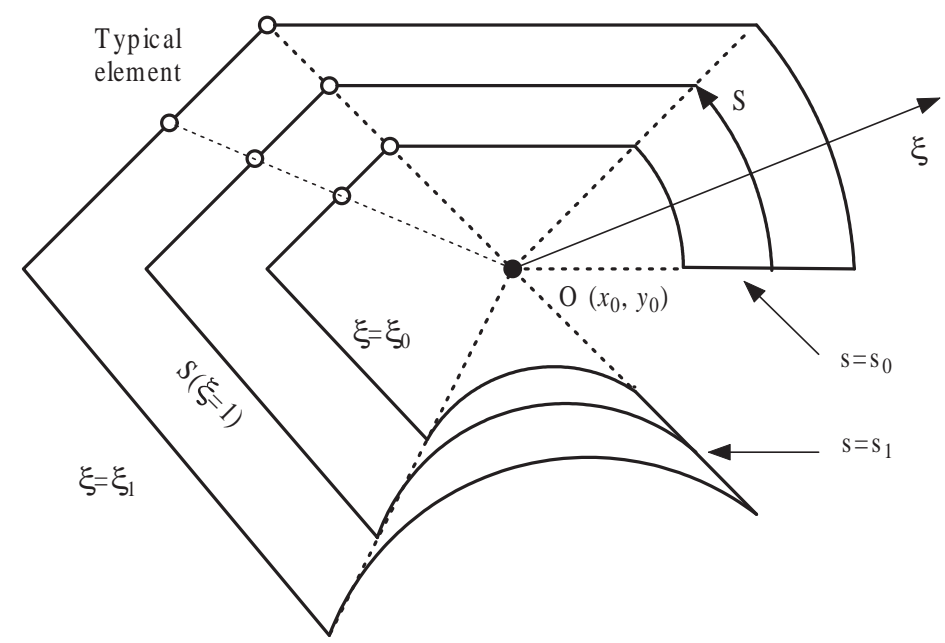

Figure 1. The coordinate definition of SBFEM.

By employing SBFEM, an approximate solution of $\phi$ is sought as

$$
\phi_{A}(\xi, s)=\mathbf{N}(s) \mathbf{a}(\xi),
$$

where $\mathbf{N}(s)$ is the shape function, the vector $\mathbf{a}(\xi)$ is analogous to the nodal values same as in FEM.

By performing scaled boundary transformation, the final scaled boundary finite-element equations are

$$
\begin{gathered}
\mathbf{q}\left(\xi_{1}\right)=\int_{S} \mathbf{N}(s)^{T}\left(\bar{v}_{n}\left(\xi_{1}, s\right)\right) \xi_{1} d s, \\
\mathbf{q}\left(\xi_{0}\right)=-\int_{S} \mathbf{N}(s)^{T}\left(\bar{v}_{n}\left(\xi_{0}, s\right)\right) \xi_{0} d s, \\
\mathbf{E}_{0} \xi^{2} \mathbf{a}(\xi)_{, \xi \xi}+\left(\mathbf{E}_{0}+\mathbf{E}_{1}^{T}-\mathbf{E}_{1}\right) \xi \mathbf{a}(\xi)_{, \xi}-\mathbf{E}_{2} \mathbf{a}(\xi)+k^{2} \xi^{2} \mathbf{M}_{0} \mathbf{a}(\xi)+\xi^{2} \mathbf{F}_{b}=\xi \mathbf{F}_{s}(\xi),
\end{gathered}
$$

where

$$
\mathbf{q}(\xi)=\mathbf{E}_{0} \xi \mathbf{a}(\xi)_{, \xi}+\mathbf{E}_{1}^{T} \mathbf{a}(\xi),
$$

and the meaning of all the coefficients can be found in $[7,10]$.

In the present study, the side-faces coincide so that the flow across the side-faces is equal and opposite, thus the term $\mathbf{F}_{s}(\xi)$ vanishes. Therefore, the final governing equation (9) is a homogeneous second-order ordinary matrix differential equation of rank $m$.

The solution of equation (9) for $\mathbf{F}_{b}=0$ is found as [8]

$$
\mathbf{a}_{1}^{S}(\zeta)=\mathbf{T H C}
$$

where matrices $\mathbf{T}$ and $\mathbf{H}$ are related to the geometry coefficients and $\mathbf{C}$ is related to the boundary conditions.

\section{Wave diffraction}

\subsection{The first-order problem}

Consider a monochromatic wave train propagating in the direction of the positive $x$ axis. A fixed vertical cylindrical structure extends from the sea bottom to the free surface of the ocean 
$\Gamma_{\infty}$

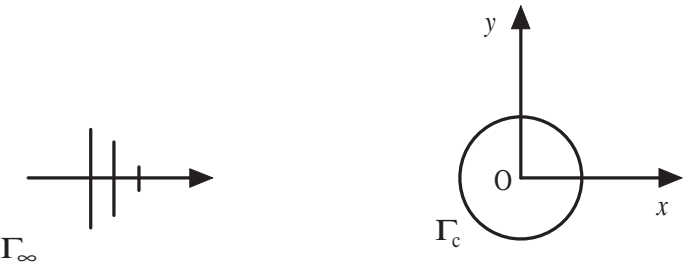

$\Omega$

$\Gamma_{\infty}$

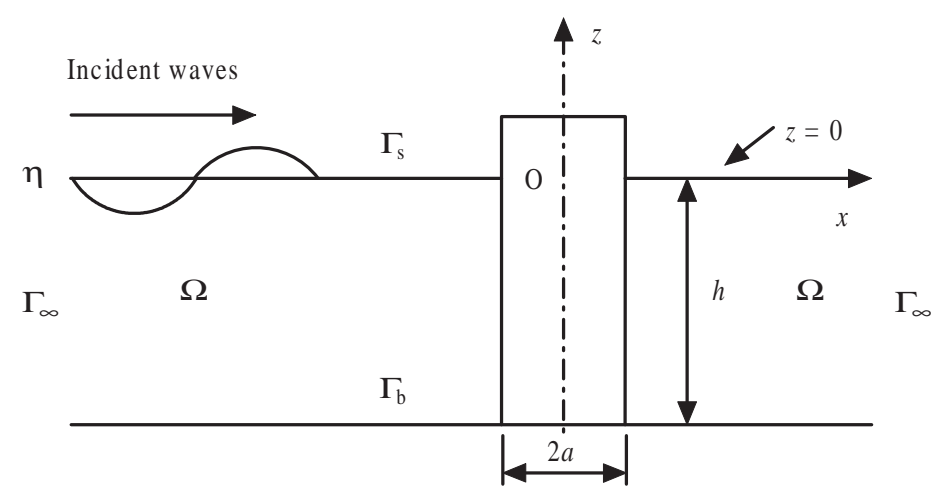

Figure 2. Definition sketch of wave diffraction by a cylindrical structure

along $z$ axis. The origin $\left(x_{0}, y_{0}\right)$ is placed at the centre of the cylinder on the mean surface level (see Fig. 2). The following notation have been used in the paper: $\Phi=$ total velocity potential, $\Phi^{I}=$ velocity potential of incident wave, $\Phi^{S}=$ velocity potential of scattered wave, $k=$ total wave number, $\omega=$ wave frequency, $h=$ water depth, $A=$ amplitude of incident wave, $a=$ cylinder radius, $t=$ time, $\rho=$ mass density of water, and $g=$ gravitational acceleration.

Assuming the fluid to be inviscid, incompressible and the flow to be irrotational, the fluid motion can be described by potential flow theory. The velocity potential of the first order incident wave is [4]

$$
\Phi_{1}^{I}=-\frac{i g A}{\omega} \frac{\cosh k(z+h)}{\cosh k h} e^{i k x} e^{-i \omega t} \quad \text { in } \quad \Omega .
$$

The first order solution of the diffraction wave was given by [8]

$$
\mathbf{a}_{1}^{S}(\zeta)=\mathbf{T H C}=-\frac{1}{k} \mathbf{T} \mathbf{H}_{h} \mathbf{T}^{-1} \overline{\mathbf{v}}_{1}^{S},
$$

where the vector $\mathbf{a}_{1}^{S}(\xi)$ is analogous to the nodal values of the velocity potential of the scattered wave, $\mathbf{T}$ is a matrix related to the coefficient matrices of the discretisation, $\mathbf{H}_{h}$ is a matrix related to the Hankel functions of the first kind, $\overline{\mathbf{v}}_{1}^{S}$ is the vector of nodal normal velocity of the first-order scattered wave on the body boundary.

\subsection{The second-order problem}

At the second order, $O\left(\epsilon^{2}\right)$, the velocity potential $\Phi_{2}$ satisfies [5]

$$
\nabla^{2} \Phi_{2}+\frac{\partial^{2} \Phi_{2}}{\partial z^{2}}=0, \quad-h<z<0,
$$

subject to the bottom condition

$$
\frac{\partial \Phi_{2}}{\partial z}=0, \quad z=-h,
$$


body boundary condition

$$
\frac{\partial \Phi_{2}}{\partial r}=0, \quad r=a,
$$

and combined free surface boundary condition

$$
\frac{\partial \Phi_{2}}{\partial z}+\frac{1}{g} \frac{\partial^{2} \Phi_{2}}{\partial t^{2}}=\frac{1}{g^{2}} \frac{\partial \Phi_{1}}{\partial t} \frac{\partial}{\partial z}\left[g\left(\frac{\partial \Phi_{1}}{\partial z}\right)+\frac{\partial^{2} \Phi_{1}}{\partial t^{2}}\right]-\frac{1}{g} \frac{\partial}{\partial t}\left[\left(\nabla \Phi_{1}\right)^{2}+\left(\frac{\partial \Phi_{1}}{\partial z}\right)^{2}\right], \quad z=0 .
$$

Eq. (17) can be simplified as

$$
\frac{\partial \Phi_{2}}{\partial z}+\frac{1}{g} \frac{\partial^{2} \Phi_{2}}{\partial t^{2}}=\mathcal{W} \cdot e^{-2 i \omega t},
$$

where the forcing function $\mathcal{W}$ is

$$
\mathcal{W}=\beta_{1} \eta_{1} \eta_{1}+\beta_{2} \nabla \eta_{1} \cdot \nabla \eta_{1},
$$

with

$$
\beta_{1}=\frac{i g k^{2}}{\omega}-\frac{3 i \omega^{3}}{g}, \quad \beta_{2}=-\frac{2 i g}{\omega} .
$$

where $\eta_{1}$ is the first order elevation without the time component.

3.3. The second-order forcing

The forcing term $\mathcal{W}$ can be split into two parts,

$$
\mathcal{W}=\mathcal{I}+\mathcal{S},
$$

where

$$
\mathcal{I}=\beta_{1} \eta_{1}^{I} \eta_{1}^{I}+\beta_{2} \nabla \eta_{1}^{I} \cdot \nabla \eta_{1}^{I},
$$

represents the self-interaction of the progressive incident wave, and

$$
\mathcal{S}=\mathcal{S}_{I S}+\mathcal{S}_{S S},
$$

with

$$
\mathcal{S}_{I S}=2\left[\beta_{1} \eta_{1}^{I} \eta_{1}^{S}+\beta_{2} \nabla \eta_{1}^{I} \cdot \nabla \eta_{1}^{S}\right],
$$

being the cross-interaction between incident and scattered waves, and

$$
\mathcal{S}_{S S}=\left[\beta_{1} \eta_{1}^{S} \eta_{1}^{S}+\beta_{2} \nabla \eta_{1}^{S} \cdot \nabla \eta_{1}^{S}\right],
$$

the self-interaction of the scattered wave.

For plane incident waves, $\mathcal{I}$ can be explicitly expressed as

$$
\mathcal{I}=\frac{3 i \omega k A^{2}}{4 \sinh k h \cosh k h} e^{2 i k r \cos (\theta)},
$$

$\eta_{1}^{I}$ and $\eta_{1}^{S}$ can be expressed for short as

$$
\eta_{1}^{I}=\sum_{m=-\infty}^{+\infty} T_{m} e^{i m \theta}, \quad \eta_{1}^{S}=\sum_{m=-\infty}^{+\infty} B_{m} e^{i m \theta}
$$


where

$$
T_{m}(r)=A i^{m} J_{m}(k r), \quad B_{m}(r)=A i^{m} \alpha_{m} H_{m}(k r),
$$

with $\alpha_{m}=-J_{m}^{\prime}(k a) / H_{m}^{\prime}(k a) . \quad J_{m}$ and $H_{m}$ are the Bessel function of the first kind and the Hankel function of the first kind respectively.

The quadratic products of $\eta_{1}^{I}$ and $\eta_{1}^{S}$ can be written in an abbreviation form

$$
\eta_{1}^{I} \eta_{1}^{S}=\sum_{m=-\infty}^{+\infty} T_{m} e^{i m \theta} \sum_{n=-\infty}^{+\infty} B_{n} e^{i n \theta}=\sum_{m=-\infty}^{+\infty} \sum_{n=-\infty}^{+\infty} T_{m-n} B_{n} e^{i m \theta}
$$

Therefore, $S$ can be expressed as a Fourier series

$$
\mathcal{S}=\sum_{m=-\infty}^{+\infty} \mathcal{S}_{m} e^{i m \theta}=\sum_{m=0}^{+\infty} \epsilon_{m} \mathcal{S}_{m} \cos m \theta
$$

with

$$
\mathcal{S}_{m}=\sum_{n=-\infty}^{+\infty}\left\{\left[\beta_{1}-\frac{n(m-n)}{r^{2}} \beta_{2}\right] B_{n}\left(2 T_{m-n}+B_{m-n}\right)+\beta_{2} \frac{\partial B_{n}}{\partial r}\left(2 \frac{\partial T_{m-n}}{\partial r}+\frac{\partial B_{m-n}}{\partial r}\right)\right\},
$$

where $\epsilon_{m}=2$ for $m \geq 1$, and $\epsilon_{0}=1$.

\subsection{Second-order Boundary-value problem}

The second-order wave potential can be decomposed as

$$
\Phi_{2}=\left(\phi_{2}^{I I}+\phi_{2}^{S S}\right) e^{-2 i \omega t},
$$

where $\Phi_{2}^{I I}$ and $\Phi_{2}^{S S}$ represent the responses to $\mathcal{I}$ and $\mathcal{S}$ respectively. Both of $\Phi_{2}^{I I}$ and $\Phi_{2}^{S S}$ satisfy governing equation (14), bottom condition (15) and body condition (16). They also satisfy the following specific free surface boundary conditions respectively

$$
\begin{aligned}
\frac{\partial \phi_{2}^{I I}}{\partial z}-\frac{4 \omega^{2}}{g} \phi_{2}^{I I} & =\mathcal{I}, \quad z=0, \\
\frac{\partial \phi_{2}^{S S}}{\partial z}-\frac{4 \omega^{2}}{g} \phi_{2}^{S S} & =\mathcal{S}, \quad z=0 .
\end{aligned}
$$

and suitable radiation conditions at infinity.

\subsection{Response to $\mathcal{I}$}

$\Phi_{2}^{I I}$ can be further decomposed as

$$
\phi_{2}^{I I}=\phi_{2}^{I}+\phi_{2}^{I S},
$$

where $\phi_{2}^{I}$ is the second-order component of the incoming wave and $\phi_{2}^{I S}$ is the diffracted wave corresponding to $\phi_{2}^{I}$. Both of them satisfy governing equation (14), bottom condition (15). Additionally, $\phi_{2}^{I}$ only satisfies the inhomogeneous free surface condition (33). The solution is the second-order component of the incoming wave, written as

$$
\phi_{2}^{I}=-\frac{3 i \omega A^{2} \cosh [2 k(z+h)]}{16 \sinh ^{4} k h} e^{2 i k r \cos (\theta)}
$$


Expression $\cosh [2 k(z+h)]$ can be expanded as

$$
\cosh [2 k(z+h)]=\sum_{n=0}^{+\infty} a_{n} \frac{\cos \left[\kappa_{n}(z+h)\right]}{\cos \left(\kappa_{n} h\right)},
$$

where $\kappa_{n}$ is the $n t h$ positive real root of $-4 \omega^{2}=g \kappa_{n} \tan \left(\kappa_{n} h\right)(n=1,2,3 \ldots), \kappa_{0}=-i \kappa$, $4 \omega^{2}=g \kappa \tanh (\kappa h)$, and

$$
a_{n}=\frac{16 \omega^{2} \kappa_{n} \sinh ^{2}(k h)}{g\left(4 k^{2}+\kappa_{n}^{2}\right)\left[\sin \left(2 \kappa_{n} h\right)+2 \kappa_{n} h\right]}
$$

Thus (36) can be written in polar coordinate as

$$
\phi_{2}^{I}=-\frac{3 i \omega A^{2}}{16 \sinh ^{4} k h} \sum_{m=0}^{\infty} a_{m} \frac{\cos \left[\kappa_{m}(z+h)\right]}{\cos \left(\kappa_{m} h\right)} \sum_{n=0}^{\infty} \epsilon_{n} i^{n} J_{n}(2 k r) \cos (n \theta) .
$$

The part $\phi_{2}^{I S}$ satisfies the homogeneous free-surface condition but the inhomogeneous condition on the cylinder

$$
\frac{\partial \phi_{2}^{I S}}{\partial r}=-\frac{\partial \phi_{2}^{I}}{\partial r}, \quad r=a,
$$

So it can be regarded as a free wave radiating energy to infinity.

The solution of $\phi_{2}^{I S}$ can be sought as

$$
\phi_{2}^{I S}=\sum_{m=0}^{+\infty} \sigma_{m}(r, \theta) \frac{\cos \kappa_{m}(z+h)}{\cos \kappa_{m} h},
$$

From Eq. (14), we have

$$
\nabla^{2} \sigma_{m}-\kappa_{m}^{2} \sigma_{m}=0, \quad m=0,1,2, \ldots, \quad r>a .
$$

Defining $\tilde{\kappa}_{m}^{2}=-\kappa_{m}^{2}$, The solution can be easily found similar to equation (11)

$$
\mathbf{a}_{2}^{\sigma}=\mathbf{T H}_{1} \mathbf{C}_{1},
$$

where $\mathbf{C}_{1}$ is determined by boundary conditions.

\subsection{Response to $\mathcal{S}$}

$\Phi_{2}^{S S}$ can also be expressed as a series of depth-eigenfunctions for frequency $2 \omega[1]$,

$$
\Phi_{2}^{S S}=-\frac{i g}{2 \omega} \sum_{m=0}^{+\infty} \zeta_{m}(r, \theta) \frac{\cos \kappa_{m}(z+h)}{\cos \kappa_{m} h},
$$

Define $f_{n}=\cos \kappa_{n}(z+h)$ and employ Green's formula, we have

$$
\int_{-h}^{0}\left(f_{n} \frac{\partial^{2} \Phi_{2}^{S S}}{\partial z^{2}}-\Phi_{2}^{S S} \frac{\partial^{2} f_{n}}{\partial z^{2}}\right) d z=\left[f_{n} \frac{\partial \Phi_{2}^{S S}}{\partial z}-\Phi_{2}^{S S} \frac{\partial f_{n}}{\partial z}\right]_{-h}^{0},
$$

Using the orthogonality of $f_{n}$, we obtain

$$
\nabla^{2} \zeta_{n}-\kappa_{n}^{2} \zeta_{n}=C_{s} \mathcal{S}, \quad n=0,1,2 \ldots
$$

where

$$
C_{s}=-\frac{8 i \omega \kappa_{n} \cos ^{2}\left(\kappa_{n} h\right)}{g\left[2 \kappa_{n} h+\sin \left(2 \kappa_{n} h\right)\right]}
$$

The solution of equation (46) can be formulated as

$$
\mathbf{a}_{2}^{\zeta}=\mathbf{T} \mathbf{H}_{1} \mathbf{C}_{2}(\xi)
$$

where $\mathbf{C}_{2}(\xi)$ is a function of the radial coordinate $\xi$ and is determined by boundary conditions. 


\subsection{Other physical quantities}

The pressure $p(r, \theta, z, t)$ at any point $(r, \theta, z)$ is determined by the Bernoulli's equation:

$$
\frac{p}{\rho}+g z+\Phi_{t}+\frac{1}{2}\left[\Phi_{r}^{2}+\frac{1}{r^{2}} \Phi_{\theta}^{2}+\Phi_{z}^{2}\right]=0
$$

Based on the perturbation theory, the first- and second-order terms of the hydrodynamic pressure are

$$
\begin{aligned}
& p_{1}=-\rho \Phi_{1 t}, \\
& p_{2}=-\rho\left[\Phi_{2 t}+\frac{1}{2}\left(\Phi_{1 r}^{2}+\frac{1}{r^{2}} \Phi_{1 \theta}^{2}+\Phi_{1 z}^{2}\right)\right],
\end{aligned}
$$

The total horizontal force per unit length in the direction of wave propagation can be determined by integrating the pressure along the cross-sectional contour at a given depth $z$. Therefore, we have

$$
\left(\frac{d F_{x}}{d z}\right)_{j}=-\left.a \int_{0}^{2 \pi} p_{j}\right|_{r=a} \cos (\theta) d \theta, \quad j=1,2 .
$$

The total horizontal force $F_{x}$ on the cylinder can be calculated by vertically integrating the $\frac{d F_{x}}{d z}$ from the seabed to the free surface. To the second-order, the upper limit of the above $z-$ integral can be taken at $z=0$ rather than $z=\eta_{1}+\eta_{2}$ as the latter would only create terms of order higher than $\epsilon^{2}$. The total horizontal force can be integrated as

$$
F_{x j}=\int_{-h}^{0}\left(\frac{d F_{x}}{d z}\right)_{j} d z, \quad j=1,2 .
$$

The second-order elevation can be obtained as

$$
\zeta_{2}(t)=\left[-\frac{1}{2 g}\left(\nabla \Phi_{1}\right)^{2}+\frac{1}{g^{2}} \frac{\partial \Phi_{1}}{\partial t} \frac{\partial^{2} \Phi_{1}}{\partial t \partial z}-\frac{1}{g} \frac{\partial \Phi_{2}}{\partial t}\right]_{z=0} .
$$

\section{Conclusions}

In this paper, second-order wave diffraction by a circular cylinder is solved by SBFEM. By splitting the forcing term into two parts, the diffraction problem is described as two secondorder boundary-value problems with different asymptotic behaviour at infinity. Both of the 3D boundary-value problems are decomposed to a number of 2D boundary-value sub-problems by separating variables in the direction of the water depth, and then are solved semi-analytically by SBFEM. Only 1D curved finite-elements are needed on the circumference of the cylinder, while the matrix differential equation in radial direction is solved completely analytically. The method can be extended to solve more complex wave-structure interaction problems resulting in direct engineering applications.

\section{Acknowledgments}

The first author is grateful for the postdoctoral fellowship from Griffith University to support this research.

\section{References}

[1] Chau F P and Eatock Taylor R 1992 J Fluid Mech 240571

[2] Ekevid T and Wiberg N-E 2002 Comput. Methods Appl. Mech. Engrg. 1913947

[3] Li S, Liang H and Li A 2008 Journal of Hydrodynamics, Ser. B 20727

[4] Mei C C 1989 The applied dynamics of ocean surface waves (Singapore: World Scientific) 
[5] Mei C C, Stiassnie M and Yue D K-P 2005 Theory and applications of ocean surface waves (Singapore: World Scientific)

[6] Song C and Wolf J P 1997 Comput. Methods Appl. Mech. Engrg. 147329

[7] Song H, Tao L and Chakrabarti S 2010 J. Comput. Phys. 2291498

[8] Tao L, Song H and Chakrabarti S 2007 Comput. Method Appl. M. 197232

[9] Teng B, Zhao M and He G H 2006 Int. J. Numer. Meth. Fluids 52659

[10] Wolf J P 2003 The scaled boundary finite element method (Chichester: Wiley) 